\section{FRI0355 \\ PREDICTIVE ABILITY OF AVAILABLE 10 YEARS CARDIO-VASCULAR RISK ALGORITHMS IN SYSTEMIC LUPUS ERYTHEMATOSUS: A RETROSPECTIVE STUDY ON 2 ITALIAN LUPUS COHORT}

D.P.E. Margiotta ${ }^{1}$, S. Fasano ${ }^{2}$, L. Navarini ${ }^{1}$, L. Pierro ${ }^{2}$, A. Riccardi², G. Dolcini ${ }^{1}$, G. Valentini ${ }^{2}$, A. Afeltra ${ }^{1} .{ }^{1}$ Unit of Immuno-Rheumatology, Universita Campus BioMedico di Roma, Rome; ${ }^{2}$ Rheumatology Unit, Università degli studi della Campania Luigi Vanvitelli, Naples, Italy

Background: Patients with Systemic Lupus Erythematosus (SLE) present an increased incidence of Cardio-Vascular Events (CVE) compared to general population, and the difference with healthy subjects is particularly evident in young SLE women.

Objectives: The aim of this study is to assess the predictive ability of established 10 years $\mathrm{CV}$ risk models in SLE

Methods: A retrospective analysis of two Italian SLE prospective cohorts was performed. SLE patients without previous CVE, with age $\geq 25$ years, a minimum continuative follow-up of 10 years and sufficient data to calculate the 10 years risk scores were enrolled. The 10 years CVE risk scores were calculated at the first observation and all CVE were prospectively recorded in the following 10 years. We calculated the following scores: the QRisk3, the Framingham CV disease 10 years score, the HeartScore (Europe Low Risk) and the SLE CV Risk Score proposed by Petri et al. Discriminatory ability for CV risk prediction was estimated by the area under the receiver operating characteristic curve. Hosmer-Lemeshov $(\mathrm{HL})$ tests was used to evaluate calibration comparing the observed versus expected number of events

Results: Analysis was performed on 131 SLE patients (mean baseline age of 37 \pm 11 years). We observed 10 CVE during the 10 years follow-up from baseline ( 3 acute coronary syndrome, 4 stroke, 1 transitory ischaemic attack and 2 peripheral artery disease). The AUC values were $0.75(95 \% \mathrm{Cl} 0.55-0.94)$ for QRisk3, 0.66 $(0.45-0.88)$ for Framingham score, $0.62(0.41-0.82)$ for the HeartScore and 0.7 $(95 \% \mathrm{Cl} 0.55-0.85)$ for the SLE CV risk score. The $\mathrm{p}$ values of $\mathrm{HL}$ test were 0.8 for Qrisk3 and SLE CV score and 0.4 for Framingham score and HeartScore, suggesting a good model fit for all the $\mathrm{CV}$ risk scores. Considering scores with better discriminative ability and calibration, $20 \%$ of CVE were observed with Qrisk3 score lower then $3.6 \%$ and with SLE CV risk score between $6 \%$ and $8 \%$. Discriminative ability and calibration were not improved by multiplying by 2 the Framingham score and the HeartScore.
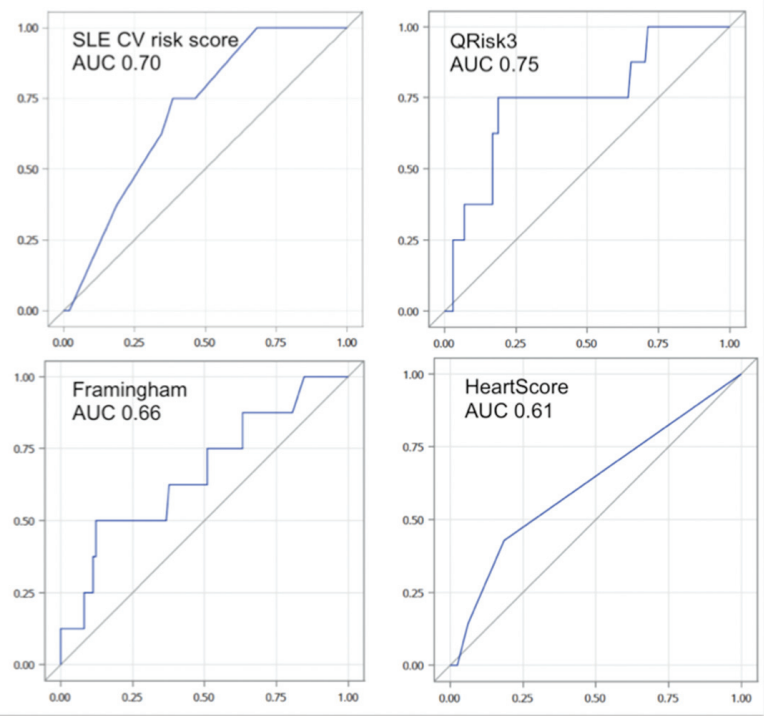

Conclusions: The available $\mathrm{CV}$ risk scores demonstrate a moderate predictive ability of 10 years CVE in SLE. We observed a better model fit for QRisk3 and SLE CV risk score. Nevertheless, a considerable proportion of patients, with very low predicted CV risk, developed CVE during follow-up.

\section{REFERENCES:}

[1] Petri MLS. Systemic lupus erythematosus Cardiovascular Risk Equation. [Abstract]. Arthritis Rheum 2012;64.

[2] Urowitz MB, Ibañez D, Su J, et al. Modified Framingham Risk factor score for systemic lupus erythematosus. J Rheumatol 2016;43:875-9.

Disclosure of Interest: None declared

DOI: 10.1136/annrheumdis-2018-eular.7445

\section{FRI0356 \\ ORGAN DAMAGE IN SYSTEMIC LUPUS ERYTHEMATOSUS IS CONSISTENTLY ASSOCIATED WITH INCREASED MORTALITY: A META-ANALYSIS}

E.R. Hammond ${ }^{1}$, I.B. Murimi ${ }^{2}$, D.H. Lin ${ }^{2}$, H. Nab ${ }^{3}$, H. Kan ${ }^{2}$, O. Onasanya ${ }^{2}$, J. C. Tierce ${ }^{2}$, X. Wang ${ }^{1}$, B. Desta ${ }^{1}$, G.C. Alexander ${ }^{2}{ }^{1}$ AstraZeneca, Gaithersburg, MD; ${ }^{2}$ Johns Hopkins Bloomberg School of Public Health, Baltimore, MD, USA; ${ }^{3}$ AstraZeneca, Cambridge, UK

Background: More than half of all patients with systemic lupus erythematosus (SLE) develop organ damage over time, including damage to the kidney, skin, car diovascular, musculoskeletal and central nervous systems. Several mechanisms have been associated with organ damage, including long-term steroid use. SLE organ damage, like comorbid disease, may contribute to increased mortality.

Objectives: We conducted a systematic literature review and meta-analysis of the association between organ damage in SLE and mortality.

Methods: A literature search (January 2000-February 2017) of PubMed, EMBASE, Cochrane Library, and Latin American and Caribbean Health Sciences Literature from four continents evaluating organ damage by the Systemic Lupus International Collaborating Clinics/American College of Rheumatology Damage Index (SDI) and mortality was conducted. Exclusion criteria included non-English language articles and study designs that did not report original, population-level measures of association. We used a random-effects meta-analysis to evaluate studies that modelled SDI as a continuous predictor of mortality and reported hazard ratios $(\mathrm{HR})$ associated with a 1-unit SDI increase.

Results: The search yielded 10420 articles, of which, 21 prospective cohort studies were selected. Ten studies evaluating SDI as a continuous variable and reporting $\mathrm{HR}$ as measure of association were pooled and meta-analysed. The pooled HR of mortality for a 1-unit increase in SDI was 1.34 (95\% confidence interval [CI]: $1.21-1.44 ; \mathrm{p}<0.001)$. A study of 213 patients followed for 13 years in China yielded the greatest risk of mortality for a 1-unit SDI increase (HR 3.65, [95\% Cl: 1.52 8.76]). When excluded from the meta-analysis, the pooled HR of mortality for a 1 unit increase in SDI was 1.32 (95\% Cl: $1.25-1.42$; $p<0.001)$. Four studies that evaluated SDI as binary variable reported $\mathrm{HR}$ for various $\mathrm{SDI}$ reference groups: $\mathrm{SDI}=0$ HR 5.10 (95\% Cl: 1.99-13.03); SDI<1: HR 3.8 (95\% Cl: 1.30-16.40); SDI<3: HR 4.74 (95\% Cl: 1.55-14.51); and SDI<5: HR 55.12 (95\% Cl: 19.15-158.63). Two studies reported odds ratios (OR) as the measure of association; for a 1-unit SDI increase, the OR was 19.7 (95\% Cl: 5.30-72.50), and for SDI=0 as reference group, the OR was 12 (95\% Cl: 1.60-92.00).

Conclusions: Organ damage in SLE is consistently associated with increased mortality across studies from various countries, regardless of how it is modelled. Novel therapies that are potentially disease modifying and steroid sparing could reduce organ damage, improve overall outcomes, and decrease mortality in patients with SLE.

Disclosure of Interest: E. Hammond Employee of: AstraZeneca, I. Murimi: None declared, D. Lin: None declared, H. Nab Employee of: AstraZeneca, H. Kan Shareholder of: GlaxoSmithKline, O. Onasanya: None declared, J. Tierce: None declared, X. Wang Employee of: AstraZeneca, B. Desta Employee of: AstraZeneca, G. C. Alexander Consultant for: Chair of the FDA's Peripheral and Central Nervous System Advisory Committee, serves as a paid consultant to IQVIA serves on the Advisory Board of MesaRx Innovations, and serves as a paid member of OptumRx's National P and T Committee. This arrangement has been reviewed and approved by Johns Hopkins University in accordance with its conflict of interest policies.

DOI: 10.1136/annrheumdis-2018-eular.2356

\section{FRI0357 VALIDATION OF A DISEASE-SPECIFIC HEALTH - RELATED QUALITY OF LIFE MEASURE FOR RUSSIAN ADULT PATIENTS WITH SYSTEMIC LUPUS ERYTHEMATOSUS: LUPUSQOL-RUSSIAN}

E. Aseeva ${ }^{1}$, L. Vorobyova ${ }^{1}$, S. Soloviev ${ }^{1}$, G. Koilubaeva ${ }^{2}$, S. Glukhova ${ }^{1}$. ${ }^{1}$ Intensive care department, V.A. Nasonova Research Institute of Rheumatology, Moscow, Russian Federation; ${ }^{2}$ rheumatology department, National Center of Cardiology and Internal Medicine named after Academician M. Mirrahimov, Bishkek, Kyrgyztan

Background: Improvements in the survival of patients with systemic lupus erythematosus (SLE) in Russia have to some extent paralleled that seen worldwide over the last 20 years, but have at times come at the cost of increased morbidity and reduction in health-related quality of Life (HRQol). Recently, a specific questionnaire to evaluate HRQol in adult SLE patients (LupusQoL) has been developed and validated in United Kingdom and in several European countries.

Objectives: To assess the validity of a LupusQoL-Russian in adult SLE patients Methods: The LupusQoL-Russian was administered to a cohort of russian patients affected with SLE. To perform a control, QoL was evaluated also with SF36. The Russian version of LupusQoL questionnaire was developed by the University of Central Lancashire and the East Lancashire Hospitals NHS Trust (www. lupusqol.com), after a linguistic validation process. Disease activity was evaluated by the SLEDAI-2K, and chronic damage by the Systemic Lupus International 